\title{
Ion Mobility Unlocks the Photofragmentation Mechanism of Retinal Protonated Schiff Base
}

N. J. A. Coughlan, B. D. Adamson, K. J. Catani, U. Wille, and E. J. Bieske* School of Chemistry, The University of Melbourne, Victoria, Australia 3010

E-mail: evanjb@unimelb.edu.au

*To whom correspondence should be addressed 


\begin{abstract}
Retinal protonated Schiff base (RPSB) is a key molecular component of biological photoreceptors and bacterial photosynthetic structures, where its action involves photoisomerization around bonds in the polyene chain. In a vacuum environment, collisional activation or exposure to visible light causes the RPSB molecule to disintegrate, producing charged molecular fragments with $m / z=248 \mathrm{Da}$, that cannot be formed by simple cleavage of the polyene chain. Photofragments resulting from laser excitation of RPSB at a wavelength of $532 \mathrm{~nm}$ are analysed in an ion mobility mass spectrometer (IMMS) and found to be the protonated Schiff base of $\beta$-ionone. Density functional theory calculations at the M062X/cc-pVDZ level support a fragmentation mechanism in which RPSB undergoes an electrocyclization/fragmentation cascade with the production of protonated Schiff base of $\beta$-ionone and toluene.
\end{abstract}

Keywords: retinal, photodissociation, electrocyclization, ion mobility, mass spectrometry 


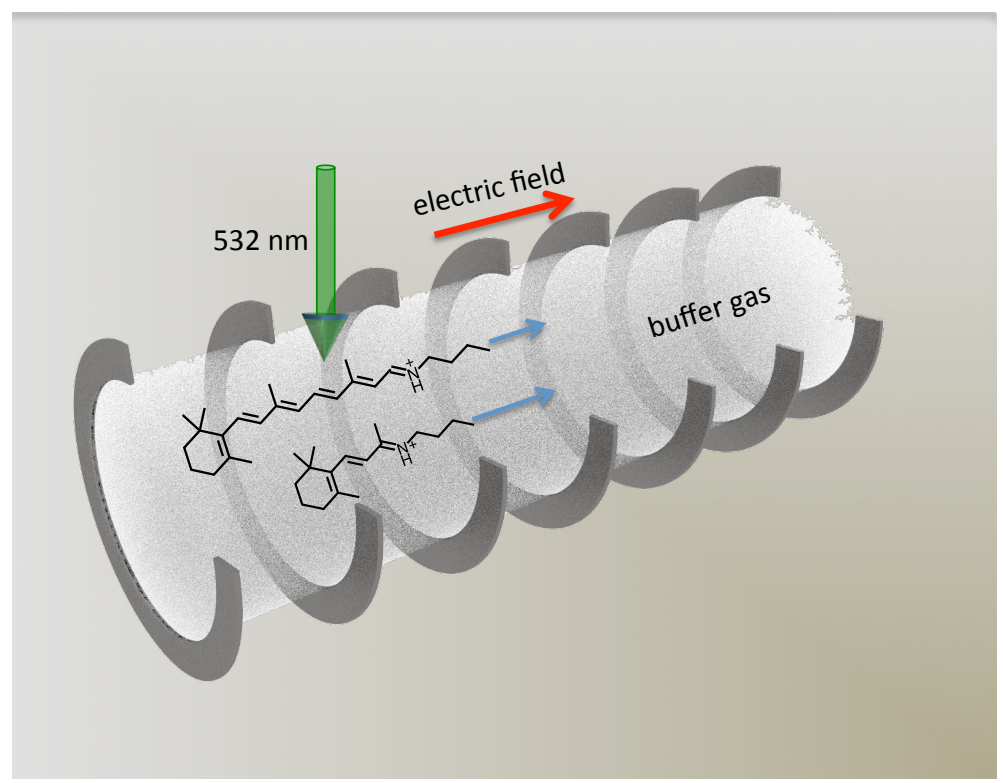

TOC Graphic: Retinal protonated Schiff base is photodissociated in an ion mobility mass spectrometer. The drift mobility of the $248 \mathrm{Da}$ fragments unambiguously identifies them as isomers of $\beta$-ionone protonated Schiff base resulting from electrocyclization of the polyene chain. 
Retinal protonated Schiff base (RPSB - Figure 1.1A) is a key molecule in the visual transduction cycle and in bacterial photosynthesis, where, in both cases, its action involves photoisomerization around bonds in the polyene chain. When embedded in a host protein, RPSB is remarkably photostable and can be repeatedly photo-excited without decomposing. However, in a vacuum environment RPSB is more fragile, and exposure to visible light or collisions with neutral gas molecules, causes its disintegration. ${ }^{1-5}$ Intriguingly, the dominant charged fragment at $m / z=248$ Da cannot be generated through a simple chain cleavage and must result from a process involving cyclization of the polyene chain. ${ }^{5}$ The previously proposed decomposition mechanism, ${ }^{5}$ illustrated in Figure 1.1, involves a sub-millisecond rearrangement through a Diels-Alder mechanism giving an energized, long-lived tricyclic structure, that disintegrates on a millisecond timescale to produce a charged bicyclic $248 \mathrm{Da}$ fragment and a toluene molecule (or an isomer thereof).

Here we consider an alternative mechanism for production of the $248 \mathrm{Da}$ fragment from RPSB. As shown in Figure 1.2, a sequential electrocylization/fragmentation process leads directly to $\beta$-ionone protonated Schiff base (Figure 1.2D) and a neutral toluene molecule. This process is analogous to the elimination of toluene and xylene from carotenes observed in mass spectrometric studies. ${ }^{6,7}$ Density functional theory (DFT) calculations at the M062X/ccpVDZ level suggest that the fragment energies for the two mechanisms are comparable $(-58$ $\mathrm{kJ} / \mathrm{mol}$ for $1 \mathrm{D}$ and $-70 \mathrm{~kJ} / \mathrm{mol}$ for $2 \mathrm{E}$ with respect to the energy of RPSB).

To examine the RPSB fragmentation process we use ion mobility mass spectrometry (IMMS) to probe the 248 Da photofragments, which have different structures (and collision cross sections) for the two mechanisms. IMMS is a versatile tool for exploring conformations of charged molecules, relying on the sensitivity of a molecule's collision cross section to its structure; under the influence of an electric field, compact molecules travel more swiftly through a buffer gas than extended, unfolded molecules. ${ }^{8-13}$ IMMS has been employed to elucidate the structures of a broad range of charged molecules and clusters including carbon and silicon clusters, ${ }^{14,15}$ carbohydrates,${ }^{16}$ peptides, ${ }^{17,18}$ and proteins. ${ }^{10}$ Recently we showed 


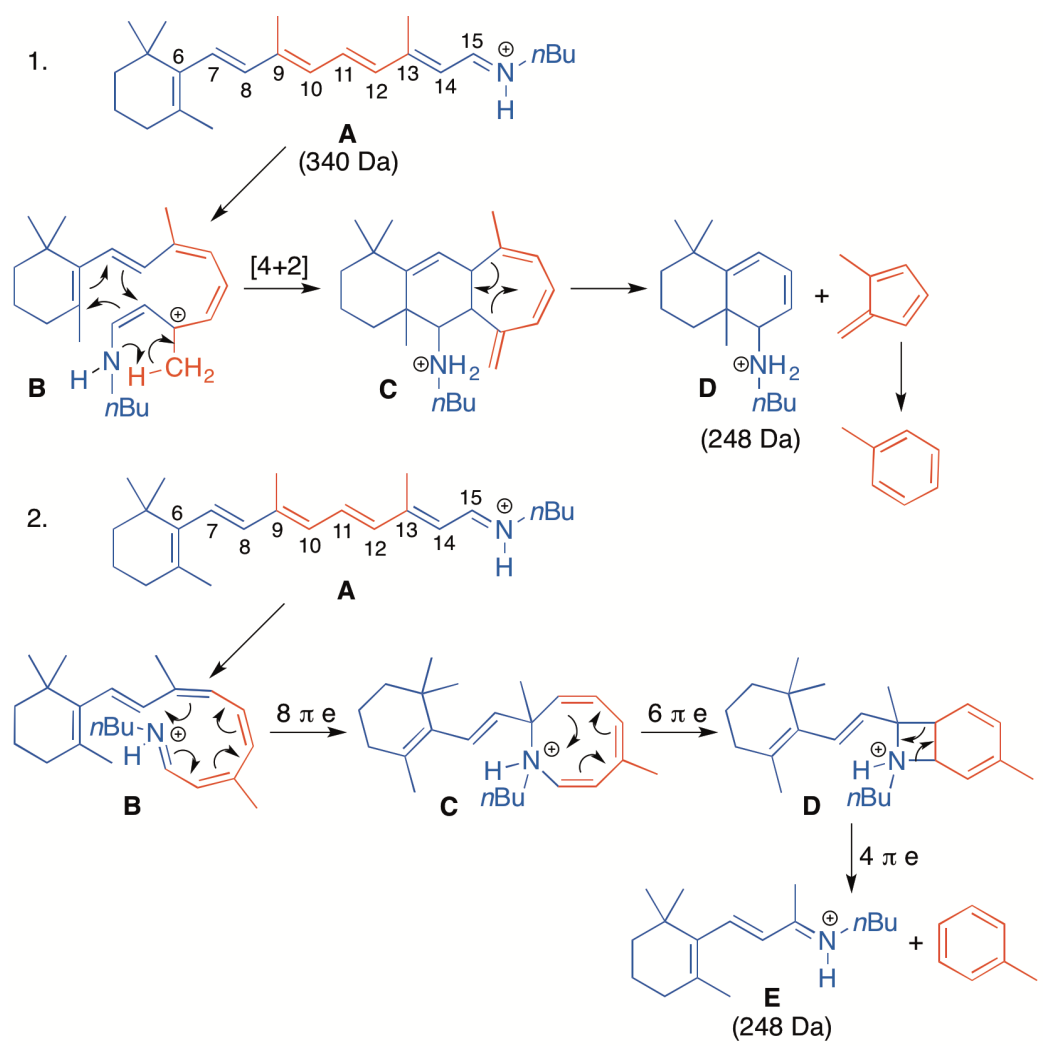

Figure 1: RPSB fragmentation mechanisms proposed by Toker et al. (1), ${ }^{5}$ and in this work (2). In mechanism 1 , RPSB undergoes a $[4+2]$ cycloaddition to form a tricyclic intermediate structure, followed by loss of toluene. In mechanism 2 , sequential $8 \pi / 6 \pi$ electron electrocyclizations lead to an intermediate containing a four membered ring, followed by elimination of toluene through a $4 \pi$ cycloreversion to give the protonated Schiff base of $\beta$-ionone. The two mechanisms involve loss of different sections of the polyene chain. 


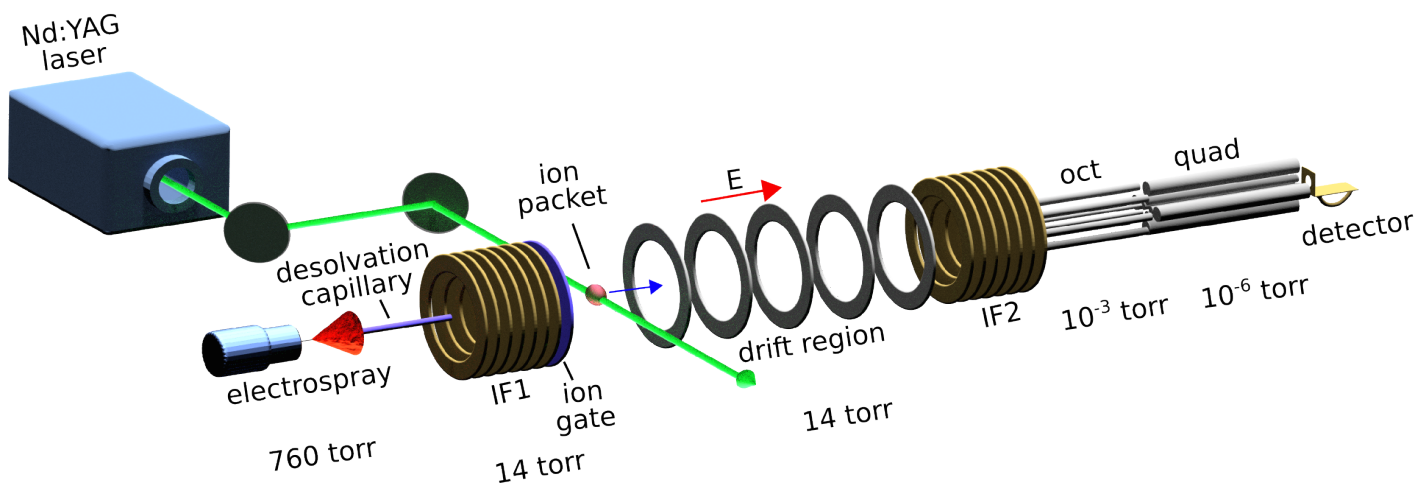

Figure 2: Ion mobility mass spectrometer for measuring collision cross sections of photofragments from RPSB. RPSB ions produced by an electrospray source pass through a heated desolvation capillary, and into an ion funnel (IF1). The ions are injected from IF1 through an electrostatic gate into the drift region filled with $\mathrm{N}_{2}$ gas $(P=14$ torr $)$ and subjected to an electric field of $\approx 44 \mathrm{~V} / \mathrm{cm}$. Here they are exposed to the pulsed output of a frequency doubled Nd:YAG laser $(532 \mathrm{~nm})$ causing photodissociation and production of 248 Da fragments. After passing through the drift tube, parent and fragment ions are collected by a second ion funnel (IF2), travel through an octopole ion guide, are mass selected by a quadrupole mass filter, and sensed by an ion detector.

that it can also be used to monitor the photoisomerization of charged molecules in the gas phase including carbocyanine dyes and RPSB. ${ }^{19-21}$ Here we demonstrate that IMMS can also be used to explore the structures of molecular photofragments and shed light on photofragmentation mechanisms.

In our experiment, RPSB ions produced by electrospray ionization are irradiated in the drift region of a purpose-built ion mobility spectrometer in which ions are propelled through $\mathrm{N}_{2}$ buffer gas by a modest electric field (Figure 2). ${ }^{19-21}$ This arrangement enables us to discriminate charged photofragments on the basis of both mass and collision cross section, providing information on their geometrical structures. If the mechanism proposed in ref. 5 prevails, one would expect to observe a relatively mobile $248 \mathrm{Da}$ bicyclic photofragment (1D in Figure 1), and perhaps a stabilized version of the $340 \mathrm{Da}$ intermediate (1C). On the other hand, the sequential electrocyclization/fragmentation reaction should yield slower monocyclic $\beta$-ionone PSB photofragments (2E in Figure 1) and possibly the stabilized intermediates $2 \mathrm{C}$ and $2 \mathrm{D}$. 


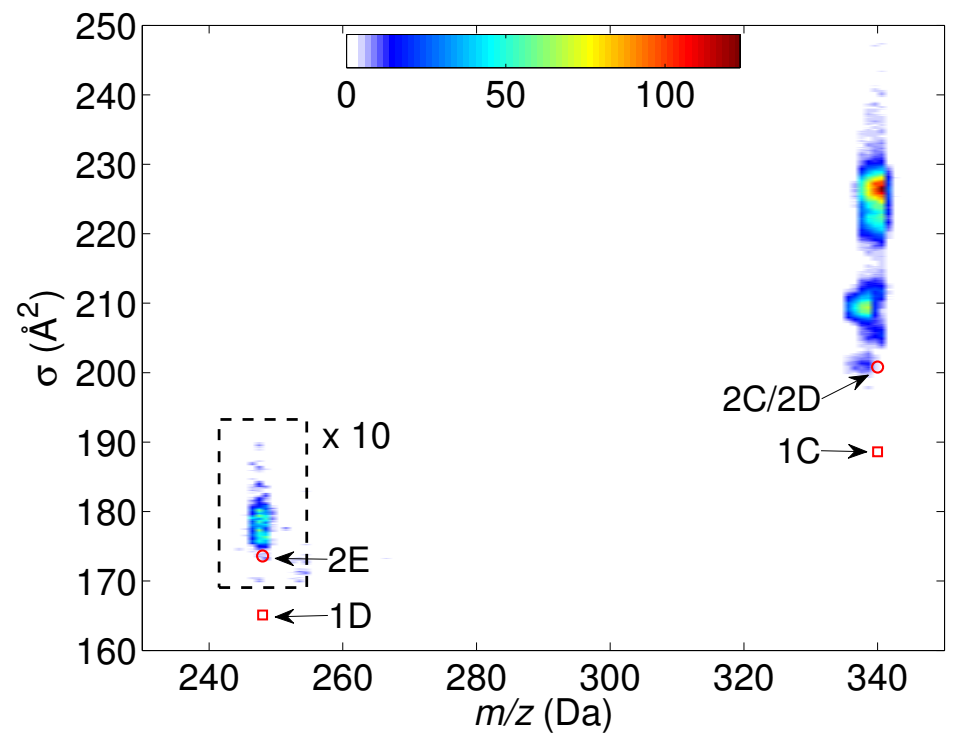

Figure 3: Laser-off ion count plotted against mass $(m / z)$ and cross section for collisions with $\mathrm{N}_{2}(\sigma)$. Red circles represent calculated data for isomers derived from the mechanism proposed in this work, whereas red squares correspond to isomers associated with the mechanism proposed by Toker et al. ${ }^{5}$ The ion count inside the dashed rectangle has been multiplied by a factor of 10 for clarity. For expanded views of this plot, see the SI.

The ion count for electrosprayed RPSB solution is plotted as a function of mass and collision cross section with $\mathrm{N}_{2}$ in Figure 3. The ion population is dominated by $340 \mathrm{Da}$, $\sigma=227 \AA^{2}$ ions associated with all-trans RPSB, but also includes 248 Da ions, as previously identified by Toker et al. ${ }^{5}$ These ions are produced in the source region of our apparatus (presumably through decomposition of RPSB ions in the electrospray desolvation capillary or first ion funnel). The ion mobility spectrum for the $248 \mathrm{Da}$ ions (obtained with the mass filter tuned to $248 \mathrm{Da}$ ) is given in Figure 4a, clearly showing that there are in fact three $248 \mathrm{Da}$ isomers with collision cross sections of $179.7,176.8$ and $167.5 \AA^{2}$, respectively. Measured collision cross sections for the dominant $248 \mathrm{Da}$ ions $\left(179.7,176.8 \AA^{2}\right)$ are closer to the predicted cross section for the monocyclic $\beta$-ionone PSB $\left(2 \mathrm{E} ; \sigma_{\text {calc }}=173.9 \AA^{2}\right.$ for the all-trans isomer) than the more compact, bicyclic structure proposed by Toker et al. ${ }^{5}$ (1D; $\left.\sigma_{\text {calc }}=166.3 \AA^{2}\right)$.

Notably, we find no evidence for the stabilized tricyclic intermediate $(1 \mathrm{C})$, which is pre- 
dicted to have a collision cross section $15 \%$ less than RPSB, and which, if it is formed rapidly and is long-lived, should be stabilized through collisions in our IMMS apparatus. Evidence for the formation of $2 \mathrm{C}$ and $2 \mathrm{D}$ is equivocal; predicted cross sections for the various stereoisomers of $2 \mathrm{C}$ and $2 \mathrm{D}$ (see $\mathrm{SI}$ ) are close to observed signal although there is no exact correspondence.

As demonstrated previously, ${ }^{21}$ when the drifting RPSB ions in our apparatus are exposed to light at low intensity $\left(\lambda=532 \mathrm{~nm}, I=1-2 \mathrm{~mJ} / \mathrm{cm}^{2} /\right.$ pulse $)$ photodissociation is minimal and instead RPSB photoisomerizes from the all-trans form to various cis forms. However, at much higher intensities $\left(I \approx 30 \mathrm{~mJ} / \mathrm{cm}^{2} /\right.$ pulse $)$ we find that 248 Da photofragments are generated from RPSB. These photofragments have a very similar ion mobility spectrum to that of the 248 Da fragments formed in the ion source (Figure 4b).

To confirm the identity of the 248 Da fragment we synthesized $\beta$-ionone PSB (Figure 1, 2E) and electrosprayed it directly into the IMMS apparatus. As shown in Figure 4c, the ion mobility spectrum for the electrosprayed sample corresponds unambiguously with the ion mobility spectra for the 248 Da fragments generated from RPSB in the ion source and through photodissociation from RPSB, conclusively identifying these fragments as $\beta$-ionone PSB. The three IMS peaks, which have slightly different relative intensities in Figure 4(ac), are presumably associated with $\beta$-ionone geometric isomers with different numbers of cis bonds along the polyene chain (calculated energies and predicted collision cross sections for $\beta$-ionone PSB isomers are given in the SI). At this stage, we assign the slowest peak to the all-trans isomer, the next fastest peak to molecules with a single cis linkage and the fastest peak to isomers with a double cis linkage.

The consecutive $8 \pi$ e and $6 \pi$ e electrocyclizations of retinal through mechanism 2 (Figure 1) are reminiscent of processes occurring in synthetic organic chemistry and in natural systems. ${ }^{22}$ To support the proposed rearrangement we used DFT M06-2X/cc-pVDZ calculations to investigate a simplified RPSB model that mimics the $\mathrm{C} 9-\mathrm{N}$ polyene chain, replacing the $\beta$-ionone and $N$-butyl substituents by vinyl and methyl groups, respectively (Figure 5 ). The 


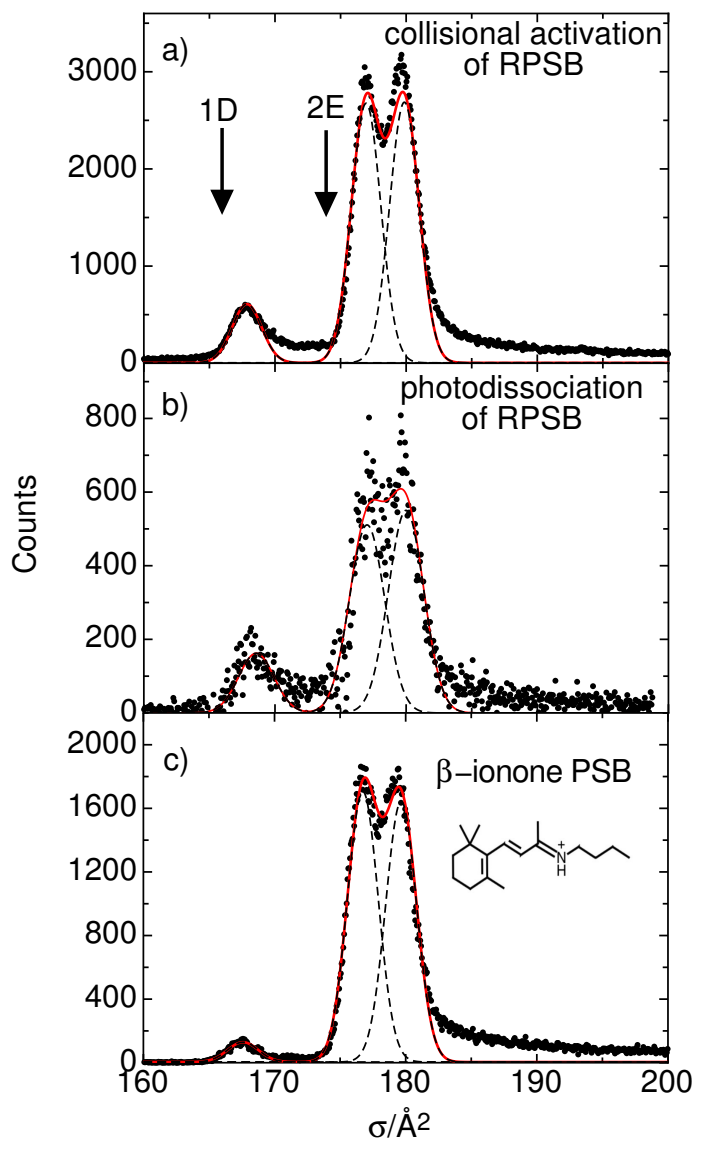

Figure 4: Ion mobility spectra for 248 Da ions formed from: (a) activation of RPSB in the ion source; (b) photodissociation of RPSB in the drift tube; (c) electrosprayed protonated Schiff base of $\beta$-ionone. In each case the peaks are fitted to 3 Gaussian functions constrained to have fwhm $=3 \AA^{2}$. Calculated collision cross sections for isomers $1 \mathrm{D}$ and $2 \mathrm{E}$ ( $\beta$-ionone) with $\mathrm{N}_{2}$ are shown in (a). 


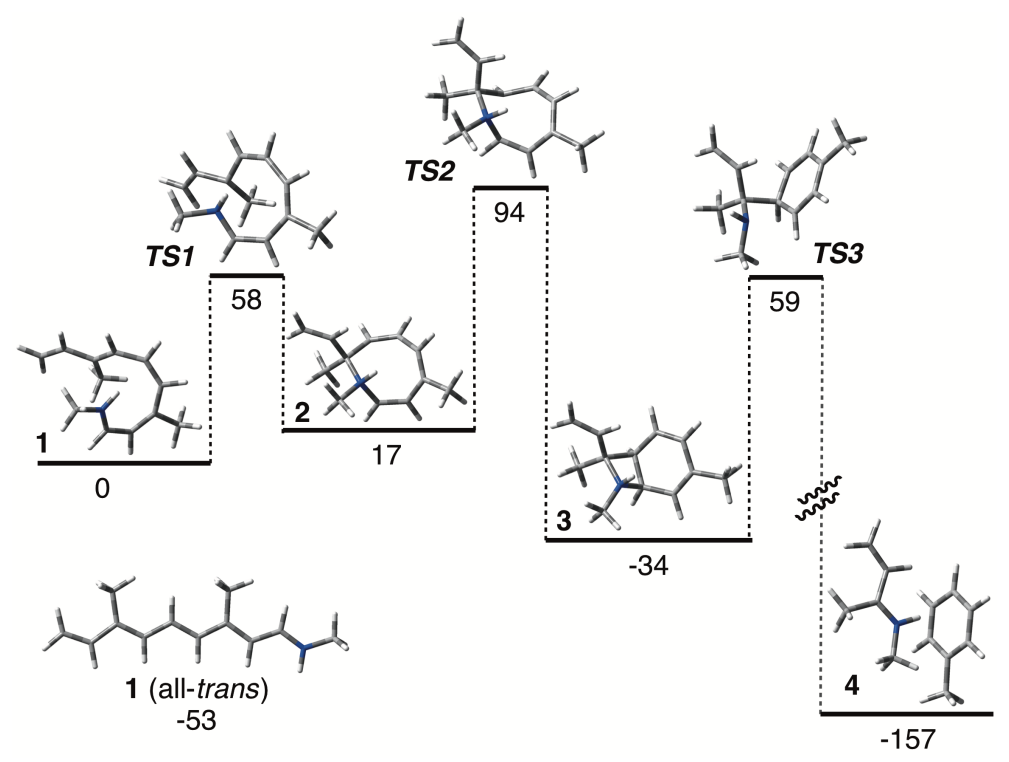

Figure 5: Calculated potential energy surface for the thermal electrocyclization/fragmentation cascade of the minimal model of RPSB. M062X/cc-pVDZ electronic energies corrected for vibrational zero point energy are in $\mathrm{kJ} / \mathrm{mol}$ and are relative to the starting molecule $\mathbf{1}$. Further stereochemical details of the process and are given in the SI.

process involves several trans-cis isomerizations to achieve the required "curled" precursor conformation for the $8 \pi$ e cyclization $(\mathbf{1})$. The reaction then proceeds in a stepwise manner, whereby the first ring closure, which leads to an 8-membered ring (structure 2) in Figure 5) is associated with a barrier of $58 \mathrm{~kJ} / \mathrm{mol}$ (TS1). The second electrocyclization has an activation energy of $77 \mathrm{~kJ} / \mathrm{mol}$ (TS2) and leads stereospecifically to a cis-bicyclo[4.2.0]octane framework (structure 3 in Figure 5). This cycloadduct is lower in energy by $34 \mathrm{~kJ} / \mathrm{mol}$ compared to starting material 1. Subsequent dissociation through cycloreversion via TS3 occurs through a concerted bond cleavage (as confirmed by Intrinsic Reaction Coordinate calculations) and produces toluene and a protonated Schiff base. Overall, the electrocyclization/fragmentation cascade is energetically highly favourable with the product association complex 4 being about $157 \mathrm{~kJ} / \mathrm{mol}$ lower in energy than the starting material $\mathbf{1}$. Mechanism 2 is possible both thermally (as shown in Figure 5) and photochemically. The only possible difference lies in the stereoselectivity of the cyclization and fragmentation steps. However, as outlined below, we believe that photoexcited RPSB rapidly internally converts from the 
$\mathrm{S}_{1}$ to the $\mathrm{S}_{0}$ state so that the photo-induced electrocyclic reaction observed under our experimental conditions occurs for highly vibrationally excited ions on the ground state PES. Under these circumstances, the stereochemical progress of the $8 \pi$ e and $6 \pi$ e electrocylizations should follow courses appropriate for thermal conditions (conrotatory for the $8 \pi$ e cyclization and disrotatory for the $6 \pi$ e cyclization). Although, the sequential electrocyclizations should be highly stereospecific processes, apart from the cis fusion of the two rings, it is not possible to predict the relative stereochemistry at the cyclobutane ring for the full 2D (Figure 1), since the outcome of the electrocyclizations depends also on the geometry of the C9-C10 and $\mathrm{C}=\mathrm{N}$ double bond in RPSB. As explained above, under our experimental conditions, we cannot unequivocally determine whether $2 \mathrm{C}$ and $2 \mathrm{D}$ are sufficiently long-lived to enable their detection, and, therefore, detailed knowledge of their stereochemistry is not essential at this stage.

It should also be noted that DFT M06-2X/cc-pVDZ computations for a simplified model system for $1 \mathrm{C}$ did not reveal a fragmentation pathway leading to $1 \mathrm{D}$. Instead, the calculations suggest that heterolytic cleavage of the $\mathrm{C}-\mathrm{NR}_{3}^{+}$bond in $1 \mathrm{C}$ is a more viable process.

The dominance of RPSB photoisomerization over photodissociation at low light levels causes us to believe that in our apparatus photodissociation of RPSB involves consecutive absorption of two or more $532 \mathrm{~nm}$ photons. ${ }^{21}$ Possibly, the process occurs through absorption of one photon followed by rapid internal conversion, absorption of a second photon, again followed by internal conversion, trans-cis isomerizations, $8 \pi$ e electrocylization, $6 \pi \mathrm{e}$ electrocylization, and dissociation, all on the ground state potential energy surface. In contrast, RPSB dissociation in the ion storage ring is believed to involve absorption of a single photon. ${ }^{5}$ The magnitude of the calculated energy barriers for the simplified model suggests that single photon photodissociation of RPSB should be possible over the 500-610 nm range, although the process may be sufficiently slow that in our experiment the reactant RPSB ions are collisionally quenched in the drift tube, where the collision rate is $\approx 5 \times 10^{8} \mathrm{~s}^{-1}$, before surmounting the initial barrier to cyclization. On the other hand, in the ion ring experiment, 
where the pressure is $\leq 10^{-10}$ torr and the collision rate is much lower, ${ }^{23}$ RPSB ions that have absorbed a single photon have much more time to rearrange, and are able to dissociate on a millisecond timescale.

In summary, we have used IMMS to probe the structure of charged molecular fragments from the chromophore RPSB in the gas phase and have unequivocally identified the $248 \mathrm{Da}$ fragment as the protonated Schiff base of $\beta$-ionone. The proposed mechanism, which occurs after both thermal and photo-excitation, involves isomerization of the polyene chain, followed by a sequential electrocyclization/fragmentation cascade that leads to elimination of toluene. Eventually, the IMMS approach described in this paper should be applicable to structural and mechanistic investigations of a broad range of gas-phase photochemical reactions, augmenting more cumbersome and expensive methods including isotopic labelling.

\section{Experimental Methods}

The ion mobility apparatus is the same as used recently to investigate the photoisomerization of carbocyanine dyes and RPSB cations. ${ }^{19-21}$ In the current study, electrosprayed RPSB cations produced from a $10^{-5} \mathrm{M}$ solution of trans RPSB in 1:1 methanol: $\mathrm{H}_{2} \mathrm{O}$ (electrospray voltage $3 \mathrm{kV}$, flow rate $5 \mu \mathrm{L} / \mathrm{min}$ ), were accumulated in an ion funnel before being launched in a $400 \mu$ s pulse into a $0.9 \mathrm{~m}$ drift tube containing $\mathrm{N}_{2}$ buffer gas ( $P=14$ torr). The electric field in the drift tube $(44 \mathrm{~V} / \mathrm{cm})$ was sustained by $90 \mathrm{ring}$ electrodes. At the end of the drift tube, the ions were collected radially by a second ion funnel before passing through a $0.3 \mathrm{~mm}$ orifice into an octopole ion guide from which they exited through a $3 \mathrm{~mm}$ orifice into a quadrupole mass filter. Ions were sensed by a channeltron detector connected to a discriminator and a multichannel scaler.

The apparatus was run at $20 \mathrm{~Hz}$, with alternate ion packets exposed to the $532 \mathrm{~nm}$ output from a pulsed frequency-doubled Q-switched Nd:YAG laser. The ions' arrival time distribution (ATD) was built up as a histogram of ion counts versus time. The mobility 
resolution of the instrument was typically $t_{d} / \Delta t_{d}=60$, which can be compared to a maximum, diffusion-limited resolution under the prevailing conditions of $120 .{ }^{19}$ Under typical operating conditions with a drift field of $44 \mathrm{~V} / \mathrm{cm}$ and $\mathrm{N}_{2}$ buffer gas pressure of 14 torr, the effective temperature of the drifting RPSB ions is predicted to be $\approx 300 \mathrm{~K}^{24}$

\section{Computational Methods}

To connect the measured collision cross sections with molecular structures, we determined equilibrium geometries for different 248 and $340 \mathrm{Da}$ isomers using DFT calculations at the M062X/cc-pVDZ level. ${ }^{25}$ Collision cross sections were determined from the calculated equi-

librium structures using the trajectory method as instituted in the MOBCAL program. ${ }^{26,27}$ Atom-atom potential energy parameters for the interaction between $\mathrm{N}_{2}$ and the colliding molecule were taken from ref. 12. Calculated energies and collision cross sections for isomers of $1 \mathrm{C}, 1 \mathrm{D}, 2 \mathrm{C}, 2 \mathrm{D}$ and $2 \mathrm{E}$ are given in the SI.

\section{Acknowledgments}

This research was supported under the Australian Research Council's Discovery Project funding scheme (Project Numbers DP110100312 and DP120100100). The computations were supported by the National Computational Merit Allocation Scheme (Project m88). We thank Professor Matthew Bush for providing a modified version of the Mobcal code with $\mathrm{N}_{2}$ collision parameters described in ref. 12, Luke Gamon for assistance with synthesis and collection of GC-MS data for the protonated Schiff base of $\beta$-ionone, and Sioe See Volaric for assistance with collection of LC-MS data. 


\section{Supporting Information Available}

Further experimental and computational information, including details for the synthesis of RPSB and $\beta$-ionone PSB, and calculated energies and collision cross sections for intermediates and products shown in Figure 1, is available as SI. This material is available free of charge via the Internet at http://pubs. acs .org/.

\section{References}

(1) Andersen, L. H.; Nielsen, I. B.; Kristensen, M. B.; El Ghazaly, M. O. A.; Haacke, S.; Nielsen, M. B.; Petersen, M. A. Absorption of Schiff-Base Retinal Chromophores in Vacuo. J. Am. Chem. Soc. 2005, 127, 12347-12350.

(2) Nielsen, I.; Lammich, L.; Andersen, L. $\mathrm{S}_{1}$ and $\mathrm{S}_{2}$ Excited States of Gas-Phase SchiffBase Retinal Chromophores. Phys. Rev. Lett. 2006, 96, 018304.

(3) Lammich, L.; Nielsen, I. B.; Sand, H.; Svendsen, A.; Andersen, L. H. Probing the Sub-Microsecond Photodissociation Dynamics in Gas-Phase Retinal Chromophores. J. Phys. Chem. A 2007, 111, 4567-4572.

(4) Rajput, J.; Rahbek, D. B.; Andersen, L. H.; Hirshfeld, A.; Sheves, M.; Altoè, P.; Orlandi, G.; Garavelli, M. Probing and Modeling the Absorption of Retinal Protein Chromophores in Vacuo. Angew. Chem. Int. Ed. 2010, 49, 1790-1793.

(5) Toker, Y.; Rahbek, D. B.; Kiefer, H. V.; Rajput, J.; Antoine, R.; Dugourd, P.; Nielsen, S. B.; Bochenkova, A. V.; Andersen, L. H. Photoresponse of the Protonated Schiff-Base Retinal Chromophore in the Gas Phase. Phys. Chem. Chem. Phys. 2013, 15, 19566-19569.

(6) Carnevale, J.; Cole, E. R.; Nelson, D.; Shannon, J. S. Chemical Ionisation Mass Spectrometry of Carotenoids. Biomed. Mass Spectrom. 1978, 5, 641-646. 
(7) van Breemen, R. B.; Dong, L.; Pajkovic, N. D. Atmospheric Pressure Chemical Ionization Tandem Mass Spectrometry of Carotenoids. Int. J. Mass Spectrom. 2012, 312, $163-172$.

(8) Lanucara, F.; Holman, S. W.; Gray, C. J.; Eyers, C. E. The Power of Ion MobilityMass Spectrometry for Structural Characterization and the Study of Conformational Dynamics. Nature Chemistry 2014, 6, 281-294.

(9) Kanu, A. B.; Dwivedi, P.; Tam, M.; Matz, L.; Hill Jr, H. H. Ion Mobility-Mass Spectrometry. J. Mass Spec. 2008, 43, 1.

(10) Wyttenbach, T.; Bowers, M. T. Intermolecular Interactions in Biomolecular Systems Examined by Mass Spectrometry. Ann. Rev. Phys. Chem. 2007, 58, 511.

(11) Pierson, N. A.; Chen, L.; Russell, D. H.; Clemmer, D. E. Cis-Trans Isomerizations of Proline Residues Are Key to Bradykinin Conformations. J. Am. Chem. Soc. 2013, 135, 3186-3192.

(12) Campuzano, I.; Bush, M. F.; Robinson, C. V.; Beaumont, C.; Richardson, K.; Kim, H.; Kim, H. I. Structural Characterization of Drug-Like Compounds by Ion Mobility Mass Spectrometry: Comparison of Theoretical and Experimentally Derived Nitrogen Collision Cross Sections. Anal. Chem. 2012, 84, 1026-1033.

(13) Shvartsburg, A. A.; Smith, R. D. Fundamentals of Traveling Wave Ion Mobility Spectrometry. Anal. Chem. 2008, 80, 9689-9699.

(14) von Helden, G.; Gotts, N. G.; Bowers, M. T. Experimental Evidence for the Formation of Fullerenes by Collisional Heating of Carbon Rings in the Gas Phase. Nature 1993, $363,60$.

(15) Hudgins, R. R.; Imai, M.; Jarrold, M. F.; Dugourd, P. High-Resolution Ion Mobility Measurements for Silicon Cluster Anions and Cations. J. Chem. Phys. 1999, 111, 7865. 
(16) Both, P.; Green, A. P.; Gray, C. J.; Šardzík, R.; Voglmeir, J.; Fontana, C.; Austeri, M.; Rejzek, M.; Richardson, D.; Field, R. A. et al. Discrimination of Epimeric Glycans and Glycopeptides using IM-MS and its Potential for Carbohydrate Sequencing. Nature Chemistry 2013, 6, 65-74.

(17) Wu, C.; Siems, W. F.; Klasmeier, J.; Herbert H. Hill, J. Separation of Isomeric Peptides Using Electrospray Ionization/High-Resolution Ion Mobility Spectrometry. Anal. Chem. 2000, 72, 391-395.

(18) Pringle, S. D.; Giles, K.; Wildgoose, J. L.; Williams, J. P.; Slade, S. E.; Thalassinos, K.; Bateman, R. H.; Bowers, M. T.; Scrivens, J. H. An Investigation of the Mobility Separation of Some Peptide and Protein Ions using a New Hybrid Quadrupole/Travelling Wave IMS/OA-TOF Instrument. Int. J. Mass Spectrom. 2007, 261, 1- 12.

(19) Adamson, B. D.; Coughlan, N. J. A.; Continetti, R.; Bieske, E. J. Changing the Shape of Molecular Ions: Photoisomerization Action Spectroscopy in the Gas Phase. Phys. Chem. Chem. Phys. 2013, 15, 9540 - 9548.

(20) Adamson, B. D.; Coughlan, N. J. A.; da Silva, G.; Bieske, E. J. Photoisomerization Action Spectroscopy of the Carbocyanine Dye $\mathrm{DTC}^{+}$in the Gas Phase. J. Phys. Chem. A 2013, 117, 13319-13325.

(21) Coughlan, N. J. A.; Catani, K. J.; Adamson, B. D.; Wille, U.; Bieske, E. J. Photoisomerization Action Spectrum of Retinal Protonated Schiff Base in the Gas Phase. J. Chem. Phys. 2014, 140, 164307.

(22) Nicolaou, K. C.; Petasis, N. A.; Zipkin, R. E. The Endiandric Acid Cascade. Electrocyclizations in Organic Synthesis. 4. "Biomimetic" Approach to Endiandric Acids A-G. Total Synthesis and Thermal Studies. J. Am. Chem. Soc. 1982, 104, 5560-5562.

(23) Andersen, J. U.; Hvelplund, P.; Nielsen, S. B.; Tomita, S.; Wahlgreen, H.; Møller, S. P.; Pedersen, U. V.; Forster, J. S.; Jørgensen, T. J. D. The Combination of an Electrospray 
Ion Source and an Electrostatic Storage Ring for Lifetime and Spectroscopy Experiments on Biomolecules. Rev. Sci. Instrum. 2002, 73, 1284-1287.

(24) Revercomb, H. E.; Mason, E. A. Theory of Plasma Chromatography/Gaseous Electrophoresis. Anal. Chem. 1975, 47, 970-983.

(25) M. J. Frisch et al., Gaussian 09 Revision A.1.

(26) Mesleh, M.; Hunter, J.; Shvartsburg, A.; Schatz, G.; Jarrold, M. Structural Information from Ion Mobility Measurements: Effects of the Long-Range Potential. J. Phys. Chem. 1996, 100, 16082-16086.

(27) Shvartsburg, A. A.; Jarrold, M. F. An Exact Hard-Spheres Scattering Model for the Mobilities of Polyatomic Ions. Chem. Phys. Letts. 1996, 261, 86-91. 


\section{University Library}

\section{- M M N E R VA A gateway to Melbourne's research publications}

Minerva Access is the Institutional Repository of The University of Melbourne

Author/s:

Coughlan, NJA;Adamson, BD;Catani, KJ;Wille, U;Bieske, EJ

Title:

Ion Mobility Unlocks the Photofragmentation Mechanism of Retinal Protonated Schiff Base

Date:

2014-09-18

Citation:

Coughlan, N. J. A., Adamson, B. D., Catani, K. J., Wille, U. \& Bieske, E. J. (2014).

Ion Mobility Unlocks the Photofragmentation Mechanism of Retinal Protonated Schiff

Base. JOURNAL OF PHYSICAL CHEMISTRY LETTERS, 5 (18), pp.3195-3199. https://

doi.org/10.1021/jz501407n.

Persistent Link:

http://hdl.handle.net/11343/52030 